DOI:

\title{
Power Flow Management and Control of Energy Storage System for Electric Vehicles in Smart Girds
}

\author{
Adel A. Abou El-Ela ${ }^{1}$, Dina M. Gado ${ }^{2}$, Tamer Fetouh ${ }^{1}$, Arafa S. Mansour ${ }^{1}$ and Sahar A. Moussa ${ }^{2}$ \\ ${ }^{1}$ Department of Electrical Engineering, Faculty of Engineering, Menoufia University, Shebin El- \\ Kom, Egypt. \\ ${ }^{2}$ Department of Electrical Engineering, Faculty of Engineering, Pharos University, in Alexandria, \\ Egypt. \\ (Corresponding author: Dina.Mohamed@pua.edu.eg)
}

\begin{abstract}
Electric vehicles (EVs) have a huge development, which lead to decrease the fuel consumptions, emissions, and help in keeping the environment clean but, this development has an impact on distribution systems operation in case of the plug-in hybrid electric vehicles (PHEVs) which may not well managed. Subsequently, it is critical to select the ideal charging/discharging methods. A fuzzy logic controller (FLC) system is proposed to decide the charging/discharging priority level of each electric vehicle (EV) based on the associated state-ofcharge (SOC) level of the battery. The fuzzy logic controller is utilized to control the electric vehicle (EV) charging/discharging process to achieve the benefits of the electricity network and electric vehicles (EVs) owners, as well. The benefit of the electricity network is related to improving the voltage profile. The simulation of a Vehicle-to-Grid (V2G) System is chosen as a case study in this paper to execute simulations. The methodology is based on plug-in hybrid electric vehicles (PHEVs). The power flow control and the Powertrain component sizing are emphatically related to their design will significantly influence the vehicle performance, cost, efficiency, and fuel economy. Subsequently, it is essential to evaluate the power flow management methodology at the Powertrain design stage in order to minimize component sizing, cost, and the vehicle fuel consumption for a given driving cycle. The simulation results show the effectiveness of the fuzzy logic controller strategy for hybrid energy storage in distributing power flow between the ultra-capacitors and the batteries.
\end{abstract}

Keywords: Voltage Distribution System; Electric Vehicles; Plug-in Categories; Smart grid; Fuzzy Logic Controller.

\section{Introduction}

Smart Grid (SG) is one of the developing intrigued in power sector nowadays. SG is an integrated system of communication and power that licenses robust bidirectional communications infrastructure with distributed computers that move forward the efficiency, reliability, quality-of-service, control, and stability of optimized power delivery. The SG is characterized by the Electric Power Research Institute (EPRI) as a power system that (a) comprises of a few automatic transmission and distribution (T\&D) systems that work in an effective, reliable, and facilitated way. (b) Controls emergency situations with 'self-healing' reactions and reacts to needs of utility and energy-market. (c) Obliges zillions of consumers and encompasses a viable communications infrastructure that enables the well-timed, adaptable, and secure a stream of information required to provide power to the developing digital economies [1].

The SG is viable system that empowers conclusion users to actively take part in energy market [2]. SG holds all the choices for generation, reserve, and guarantees reliable quality for the internet thrift. SG is classified into three domains: Smart system, Smart Management System, and Smart Protection System, respectively [3]. Recently, EVs have contributed toward grid support in SG. EVs operate in two modes: (a) Consumers and (b) Prosumers. In the consumer's mode of operation, battery charging current is expended from utility and in prosumers mode, EVs infuse power to the grid at the essential time specified by the showcase operator or depending on the grid loading, such as softly stacked or intensely stacked. Likewise, the energy rates are moreover affected by previously mentioned modes of operation. The consumer's mode is suggested as Grid-to-Vehicle

ERJ, PART 1, Elec. Eng., Vol. 44, No. 3, July 2021, pp. 263 - 271 
$(\mathrm{G} 2 \mathrm{~V})$ and prosumers mode is meant as Vehicle toGrid (V2G). Within the G2V mode, EV carries on as electrical load and $\mathrm{V} 2 \mathrm{G}$ mode is acting like injecting source. Prosumers cannot store energy from EVs straightforwardly, but V2G utilizes the stored energy from battery bank of EVs back to SG, thus earning the income through power export. The charging battery is dealing with G2V mode and, the grid bears the energy demand from the parking area [4].

The rule based strategy is one of the most appropriate and adaptable to resolve assortment of issue is a fuzzy logic controller. Fuzzy logic controller is based on instinctive control approach and able to interpret human information and experience into comparing results. This makes fuzzy logic controller reasonable for complex systems and gives an effective control which can be utilized in real time application.

The main objective of this research is to design a robust, fast, and smart controller that can handle the signals coming to it from the EV and make an adjustable choice that maintains the stability of the system and increment its productivity. The control management strategies are designed using a fuzzy logic toolbox within MATLAB while the vehicle is modeled in SIMULINK.

The contributions of this paper can be summarized as follows:

- Description of the types of vehicles available according to the capacity of the batteries are presented. - Studying an integrated system that contains a load that derives its strength from more than one source is presented.

- The extent to which the vehicle during the charging and discharging process affects the stability of the system is introduced.

- A FLC is designed to control the charging and discharging process of the system.

This paper is organized as follows in section 2, the electric vehicle is discussed. Vehicle to grid (V2G) interactions in SG is introduced in section 3. In section 4 , the design of electric vehicle model is described. The fuzzy logic controller strategy and the simulation results are introduced. The optimization and power management algorithm are introduced in section 5 . Finally, the conclusions are collected in section 6 .

\section{Classification of Electric Vehicles}

Considering customer strengthening mindfulness and social acceptance of EV innovations, the demand for EVs is exponentially expanding. The dual batteries mode of EVs with storage capacity and mobile charging are conceivable for consumers and prosumers to completely take part in smart grid (SG) system [5].

The scientific categorization of electric vehicles (EVs) is illustrated in Figure 1 [6], [7] that can be classified into three types:

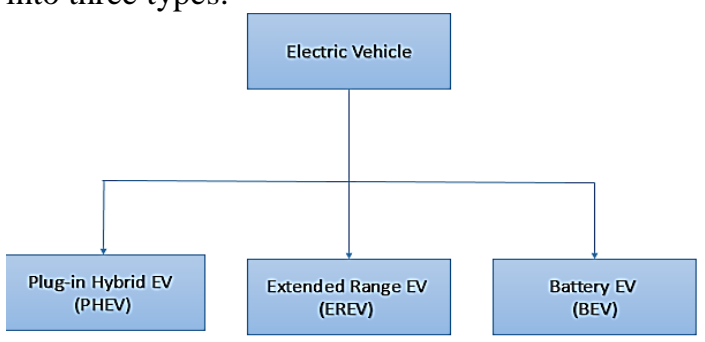

Figure 1- Electric Vehicle (EV) - Scientific Categorization.

\subsection{Plug-in Hybrid Electric Vehicle (PHEV)}

In this category, electric extend is exceptionally constrained. A little battery of 5-10 kWh capacity and charge power of $1-3 \mathrm{~kW}$ is sufficient [6]. Figure 2 depicts the sustainable energy in detail. The Energy Storage System (ESS) for existing PHEVs is electrochemical. ESS composites battery cells, bundling, battery management system (BMS), and thermal management system (TMS).

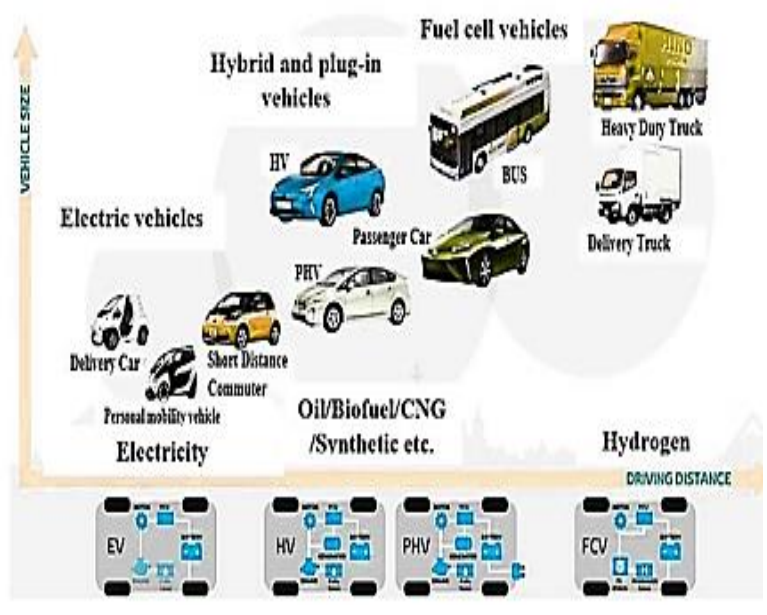

Figure 2-Roadmap towards sustainable mobility.

\subsection{Extended Range Electric Vehicle (EREV)}

This category of EV has an expanded range. Extended Range Electric Vehicle (EREV) possesses medium battery that store 10-20 kWh capacity and charge power of $6 \mathrm{~kW}$ [7]. EREVs are planned with dual power sources, battery capacity, and liquid petroleum fuel that are utilized as an inner combustion motor. EREVs are different from PHEV as they utilize a huge capacity battery to provide an endless electric extend. Besides, they require plugging the control into the network to totally charge the battery.

The fuel engine produces the driving control for the automobile and restores the vehicle battery charge. The battery in PHEV is the most part littler to estimate 
than EREV batteries as the motor of the gasoline/vehicle, generator that is used for standby its control [8].

\subsection{Battery Electric Vehicle (BEV)}

Battery Electric Vehicle (BEV) has a differing electric range of a huge battery that is greater than $20 \mathrm{kWh}$ and charge power more than $6 \mathrm{~kW}$ [7]. The BEV is completely powered by the energy storage system (ESS) of the vehicle. Today, the investigation has been done on renewable energy sources (RES) to utilize it for charging of EVs.

\section{Vehicle to Grid (V2G) Interactions in SG}

$\mathrm{V} 2 \mathrm{G}$ innovation is a system that possessing the capability of controlled bi-directional electric energy stream from the G2V for charging of the battery, and at times when the grid is requiring energy the power flows in another direction from $\mathrm{V} 2 \mathrm{G}$ giving muchneeded power at peak power consumptions [9]. EVs penetration will increase the burden on the current power system, in this way opens a plausibility of executing V2G [6].

$\mathrm{V} 2 \mathrm{G}$ is the energy management of utility load by aggregators through communication between the customers and the power grid. Three modern innovations EV are: (a) Vehicle-to Vehicle, (V2V), (b) Vehicle-to-Home V2H and (c) Vehicle-to Grid (V2G). An overview of a typical $\mathrm{V} 2 \mathrm{G}$ device is shown in Figure 3.

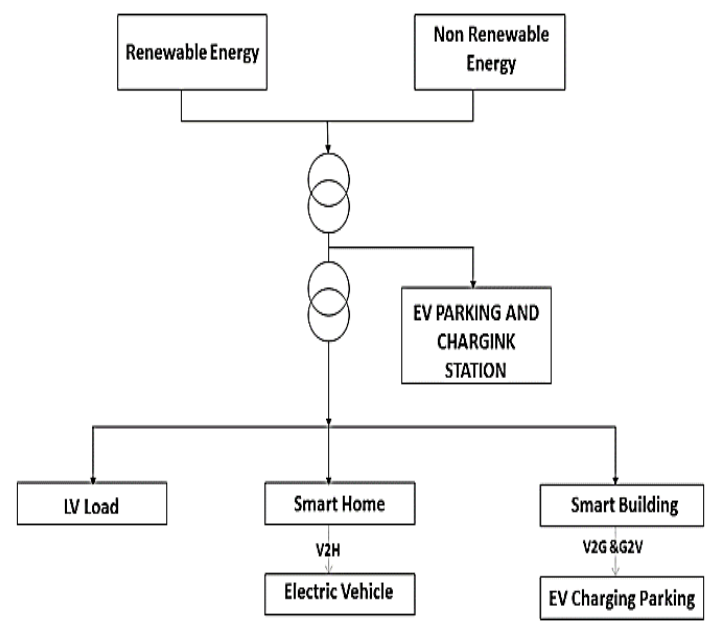

Figure 3-V2G system framework.

\subsection{V2G Power Flow}

The V2G power stream is accomplished by the nearby aggregator through communication between the grid and the consumers/prosumers. A complete data power exchange is sent straightforwardly from the smart meter to data centers. The Power streams from G2V to charge the battery and power stream in inverse to provide peak load shaving or concept of "Spinning reserve". The power flow can be in any of two modes of operation to be specific: (a) Unidirectional, and (b) bi-directional [8].

\subsubsection{Unidirectional V2G}

Unidirectional V2G controls the EVs battery charging time in one direction of power stream between power and EV. The implementation of the unidirectional $\mathrm{V} 2 \mathrm{G}$ is inexpensive and easy to control utilizing the basic command to manage the load current. Unidirectional V2G mode delivers secondary services to organize such as power system regulation and spinning reserve capacity as displayed in Figure 4.

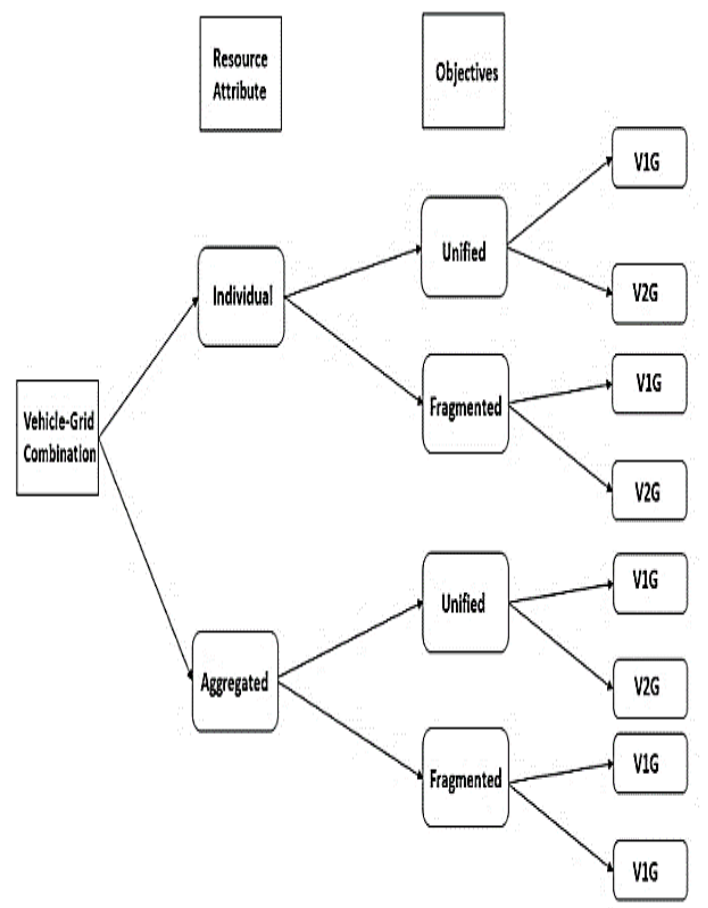

Figure 4-Power Flow Interaction.

The major administrations that cannot be obtained with the V2G unidirectional mode of operation are capping of crest loads, responsive power support, frequency regulation, and voltage regulation [9].

\subsubsection{Bi-directional V2G}

Bi-directional $\mathrm{V} 2 \mathrm{G}$ is the stream of power within the two directions; V2G and G2V accomplishing most extreme benefits. A normal EV charger comprises of bi-directional converters, AC/DC and DC/DC converters. Rectification purpose is to convert $\mathrm{AC}$ power grid to $\mathrm{DC}$ power for charging of $\mathrm{EV}$ and invert the $\mathrm{DC}$ current into $\mathrm{AC}$ before injection into the electricity network within the discharging mode. Opposite, choppers are used to control the bi- 


\section{Adel A. Abou El-Ela, Dina M. Gado, Tamer Fetouh, Arafa S. Mansour and Sahar A. Moussa "Power Flow Management and Control ......."}

directional power flow control. The DC/DC converter as a Buck converter, boost converter, and buck boost converter reacts during loading mode and discharge mode. The bidirectional V2G delivers greater flexibility and opportunities to improve the power system operations as shown in Figure 5.

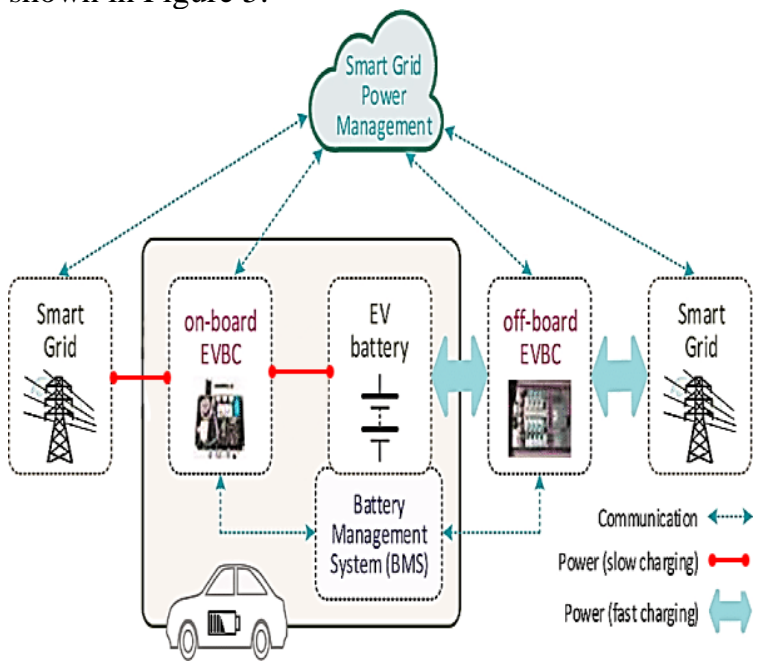

Figure 5-Bi-directional power flow between Grid and EV.

The most preferences incorporate: (a) active support of the network, (b) support of reactive power, (c) control power factor, and (d) support for the integration of RES. The support of $\mathrm{V} 2 \mathrm{G}$ two-way active power provides peak shaving services and load balancing. These administrations can be gotten by charging the EV during periods of inertia and add additional EV energy within the power grid in peak hours separated from supporting the arrangement of effective power. Bi-directional V2G has the capability of giving reactive power to guarantee the voltage regulation. The benefit can be executed with a sufficient dimensioning of the load circuit capacitor and a corresponding control circuit. The power factor control is also one of the premium services of bi-directional V2G technology that decreases the power dissipation within the grid [10], [11].

\section{Simulation Case Study \\ 4.1. System model description}

The smart grid is apportioned into four fundamental parts:(a) A diesel generator, (b) A PV farm solidified with a wind farm to deliver renewable energy, (c) A V2G system presented, and (d) finally the power grid load. The size of the smart grid shows a group of a thousand family units speaking to a low utilization day in a year. There are 100 EVs within the base model suggest that there's a 1:10 proportion between the households and EV. The single line diagram of the proposed conspire is shown in Figure 6.

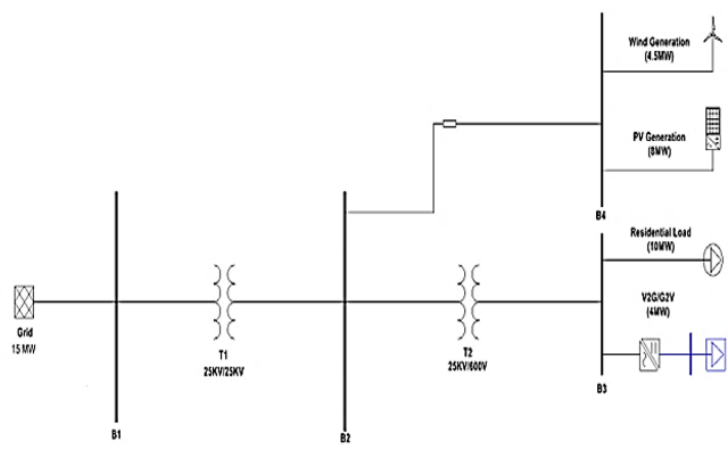

Figure 6- Single line diagram of the system.

\subsubsection{Diesel Generator}

The diesel generator equalizes the produced and consumed power. The frequency deviation of the power grid can be decided by observing the rotor speed of its synchronous machine.

\subsubsection{Renewable Energy}

There are two fundamental sources of renewable energies into the smart grid. To begin with, a PV farm produces energy comparing to three variables: (a) span of the area secured by PV farm, (b) solar panels capability, and (c) the radiance information. Secondly, a progressed model of a wind farm produces electrical energy that having a direct relation with wind velocity. When the wind accomplishes a maximum rated value, the wind farm conveys the maximum rated power.

\subsubsection{Vehicle-to-Grid (V2G)}

The V2G is taken after two fundamental functions: (a) controlling the stored charge in the batteries, and (b) utilizations of stored energy for power grid regulation [12].

\subsubsection{Consumer Load}

The Customer stack comprises of residential load and an asynchronous machine utilized to mean the heavy inductive burden (like a ventilation system) effect on the smart grid. A square relation between the mechanical torque and rotor speed, controls the asynchronous machine.

\subsection{Performance Evaluation}

The phasor mode of specialized power system allows a fast simulation of a 24-hour scenario. A normal distribution is taken after by the solar power, whereas the most raised power came at $1 \mathrm{PM}$ to $2 \mathrm{PM}$ whereas the wind shifts unrealistically all through the day and has frequent ups and downs. The utilization is low all through the day and rises to its peak during the evening, and slowly reduces during the night. Three events will impact the frequency of the grid during day time: (a) The asynchronous machine kick-off earlier at the third hour, (b) A fractional shading at twelve aggravating the solar power generation, and (c) Wind farm tripping at $22 \mathrm{hr}$ when the wind outperforms the maximum permitted wind power [13]. 


\subsection{Fuzzy Logic Controller Design}

The design comprises of four main conceptual blocks which are fuzzifer, defuzzifier, fuzzy inference system (FIS), and fuzzy rule based [12] as shown in Figure 7. In this paper, multiple input multiple output (MIMO) fuzzy logic controller is utilized. The inputs are error signal (Uerr), load required power (Pload), square of ultracapacitor power (Ucap) ${ }^{2}$ and square of speed $(\mathrm{V})^{2}$, affect the batteries in term of discharge power and charge power. For fuzzy inference system is shown in Figure 7 [14] , [15]

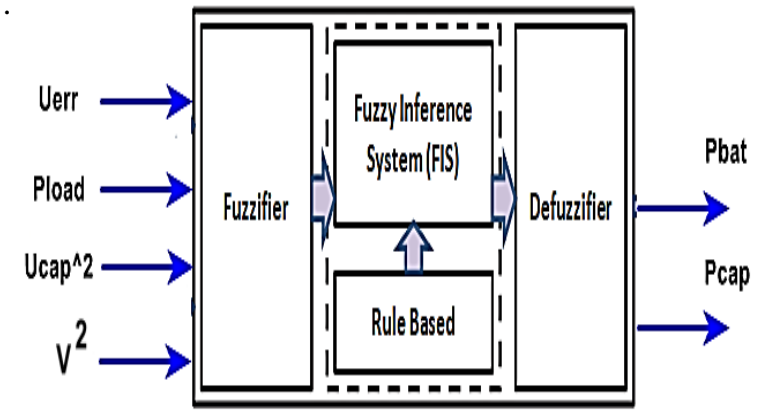

Figure 7-Fuzzy Logic Controller Scheme.

The energy management for hybrid electric vehicle (HEV) using FLC is developed using SIMULINK environment. The FLC is divided into discharge FLC and charge FLC. .The developed model is shown in Figure 8

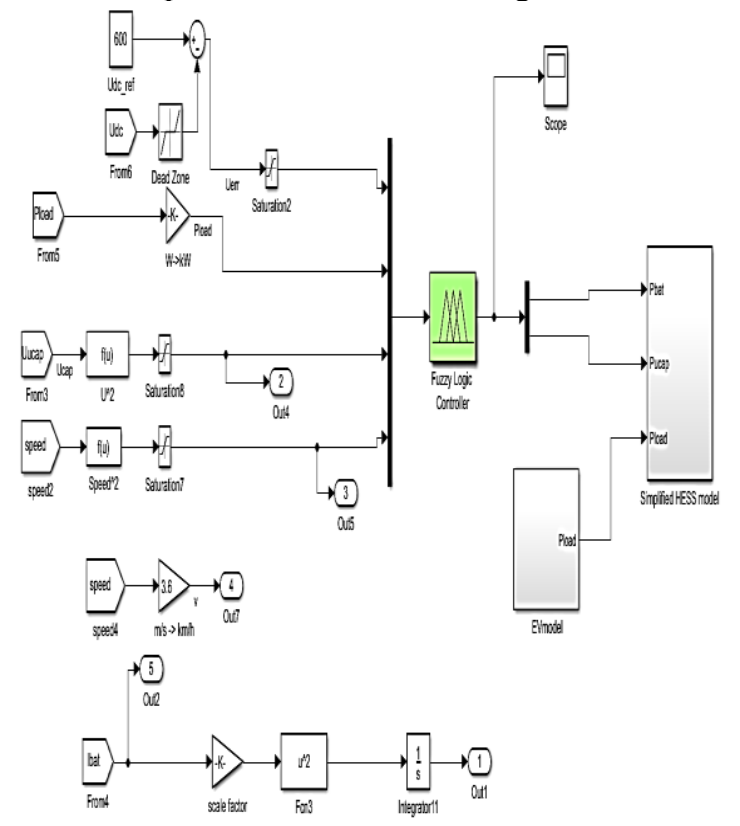

Figure 8-Power Controller Using FLC.

Discharge fuzzy logic controller input and output variables were defined as follows: first input consists of Five membership functions namely negative large $\left(n e g_{L}\right)$,
Negative small $\left(\right.$ neg $\left._{S}\right)$, zero, positive small $\left(\operatorname{pos}_{S}\right)$, And positive large $\left(\mathrm{Pos}_{L}\right)$ while second input load required power (Pload) consists of four membership functions namely Low (L), medium (M), high $(\mathrm{H})$, and very high (VH) while third input is square of ultra capacitor power $(\mathrm{Ucap})^{2}$ consists of seven membership functions namely under $(\mathrm{U})$, very low $(\mathrm{VL})$, Low $(\mathrm{L})$, medium $(\mathrm{M})$, high $(\mathrm{H})$ , very high $(\mathrm{VH})$, and over $(\mathrm{O})$ while fourth input is square of speed $(\mathrm{V})^{2}$ consists of four membership functions namely zero, low $(\mathrm{L})$, high $(\mathrm{H})$, and max. The first output variable, power of battery(Pbat) consists of three membership functions, namely charge (ch), discharge (dis), and overcharge (ovch), while the second output ultracapacitor power (Pcap) consists of two membership functions namely charge (ch) and discharge (dis). The types of membership functions are studied as shown in Figure 9. The constructed fuzzy rules matrix is based on the knowledge and intuition of hybrid electric vehicle control as presented in Table 1[16].
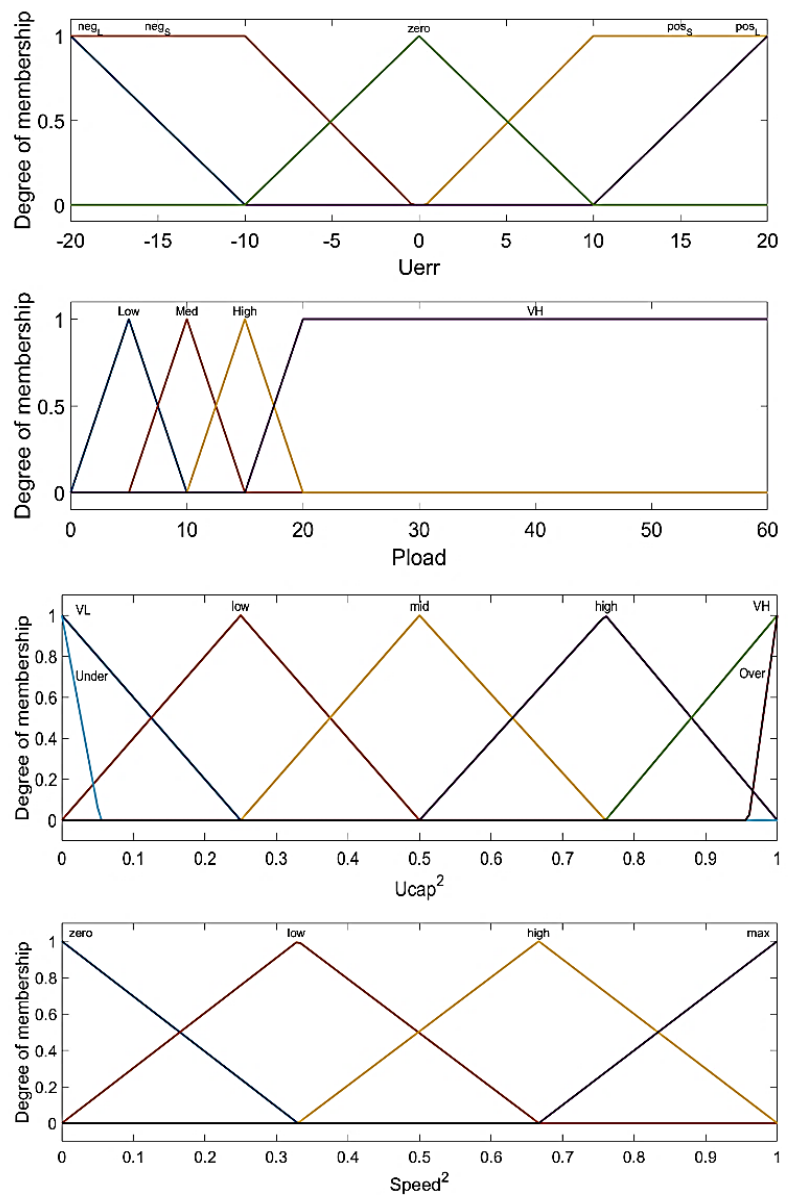

Figure 9-Fuzzy Logic Controller Outputs Membership Functions 
Table 1- Rule based on fuzzy logic controller.

\begin{tabular}{|c|c|c|c|c|c|}
\hline \multicolumn{5}{|c|}{ Inputs } & \multicolumn{2}{c|}{ Outputs } \\
\hline $\mathrm{U}_{\text {err }}$ & $\mathrm{P}_{\text {load }}$ & $\mathrm{U}_{\text {cap }}{ }^{2}$ & $\mathrm{~V}^{2}$ & $\mathrm{P}_{\text {bat }}$ & $\mathrm{P}_{\text {cap }}$ \\
\hline$n e g_{L}$ & $\mathrm{~L}$ & $\mathrm{U}$ & zero & Ch & ch \\
\hline$n e g_{S}$ & $\mathrm{M}$ & $\mathrm{VL}$ & $\mathrm{L}$ & Dis & dis \\
\hline $\operatorname{pos}_{S}$ & $\mathrm{H}$ & $\mathrm{L}$ & $\mathrm{H}$ & Ovch & \\
\hline $\operatorname{pos}_{L}$ & $\mathrm{VH}$ & $\mathrm{M}$ & $\mathrm{max}$ & & \\
\hline & & $\mathrm{H}$ & & & \\
\hline & & $\mathrm{VH}$ & & & \\
\hline & & $\mathrm{O}$ & & & \\
\hline
\end{tabular}

\subsection{Mathematical models of proposed technique}

\subsubsection{Fuzzification methods}

Given two fuzzy values $\mathrm{x}$ and $\mathrm{y}$, we characterize the taking after operations:

$(x$ and $y)=\min (x, y)$

$(\mathrm{x}$ or $\mathrm{y})=\max (\mathrm{x}, \mathrm{y})$

Not $\mathrm{x}=1-\mathrm{x}$

$(\mathrm{x}$ implies $\mathrm{y})=\max (\mathrm{x}, \mathrm{y})$

The function fuzzyset is similar to the piecewise function with the following differences:

i. The form of the calling sequence is FuzzySet $\left(x 0 x_{1}, y_{1}\right.$ , $\left.x 0 x_{2}, y_{2}, \ldots . ., x 0 x_{N}, y_{N}\right)$, where 0 may be one of $\mathrm{x}_{1}, \mathrm{x}_{2}, \ldots . . \mathrm{x}_{3}<,=$, or $<=$ and the values $\mathrm{x}_{1}, \mathrm{x}_{2}, \ldots . ., \mathrm{x}_{\mathrm{N}}$ the degree of membership functions must be in order.

ii.FuzzySet $\left(\ldots, x<x_{N}, f_{N}(\mathrm{x}), x=x_{\mathrm{n}+1}, y_{n+1}, \ldots\right)$ Interpolates the interval $\left[x_{N}, x_{N+1}\right]$ With a linear function connecting the points.

iii. The names of the constructors $\Gamma, L, \Lambda$, and $\pi$ are chosen such that the shape of the letter represents the object being represented.

Where, $\Gamma$ : generates a fuzzy set for which the membership function is monotonically increasing.

$L$ : generates a fuzzy set for which the membership function is monotonically decreasing.

$\Lambda$ : generates a fuzzy set for which the membership function achieves a maximum at a point and decrease to zero on both sides.

$\pi$ : generates a fuzzy set for which the membership function achieves a maximum on an interval and decrease to zero on both sides.

iv. The constructor Partition takes a sequence of three or more ordered points and creates a lzambda fuzzy set for each set of three consecutive points. Each of these generated fuzzy sets satisfies:

$\mu_{A_{i}}\left(x_{i}\right)=0$

$\mu_{A_{i}}\left(x_{i}+1\right)=1$
$\mu_{A_{i}}\left(x_{i}+2\right)=0$

\subsubsection{Defuzzification Methods}

Defuzzification is the process of converting a fuzzified output into a single crisp value with respect to a fuzzy set. The defuzzified value in FLC represents the action to be taken in controlling the process. The following are the known methods of defuzzification, Center of Sums (COS) Method, Center of gravity (COG) Method / Centroid of Area (COA) Method, Center of Area / Bisector of Area (BOA) Method, Weighted Average Method and Maxima method. In this paper, the Center of Sums (COS) Method is used. however, this is the most commonly used defuzzification technique. In this method, the overlapping area is counted twice. The defuzzified value $\boldsymbol{x}^{*}$ is defined as :

$$
\boldsymbol{x}^{*}=\frac{\sum_{i=1}^{N} x_{i} \sum_{k=1}^{N} \mu_{A_{k}}\left(x_{i}\right)}{\sum_{i=1}^{N} \sum_{k=1}^{N} \mu_{A_{k}}\left(x_{i}\right)}
$$

Where, $\mathrm{n}$ is the number of fuzzy sets, $\mathrm{N}$ is the number of fuzzy variables, $\mu_{A_{k}}\left(x_{i}\right)$ is the membership function for the k-th fuzzy set.

The power distribution between sources appears, according to the Requirements outlined in the preceding segment.

For each cycle, which begins and ends with the same vehicle state (i.e. The same vehicle speed and predicted slope) an average UCs power over the cycle is zero. Therefore the total load and battery capacity are almost equal as shown in Figure 10. The disparity derives from the lack of control in the hybrid source [17].

The power and torque is greatly improved after using FLC, the output speed and power is shown in Figure 11.

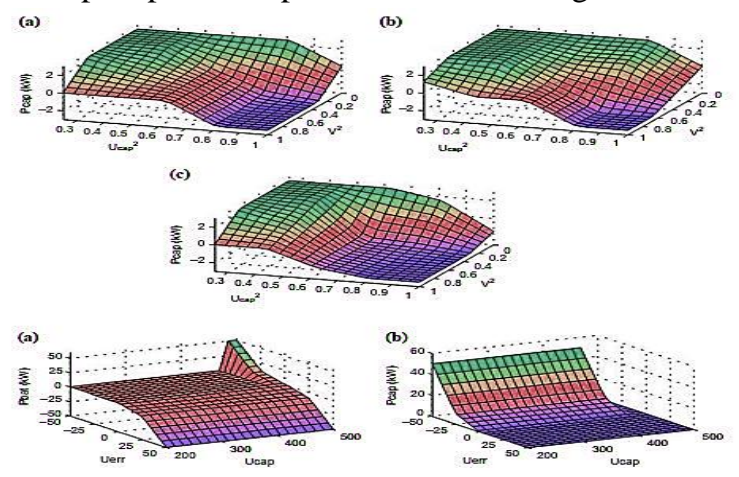

Figure 10-Relationships Between $U_{c a p}^{2}, \mathrm{~V}^{2}$ and $\mathrm{P}_{\text {cap }}$ in Different Cases. 
(a)

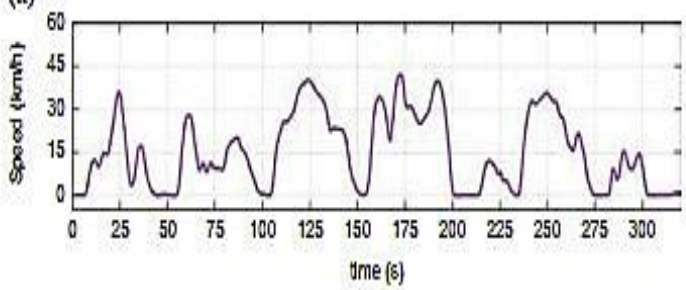

(b)

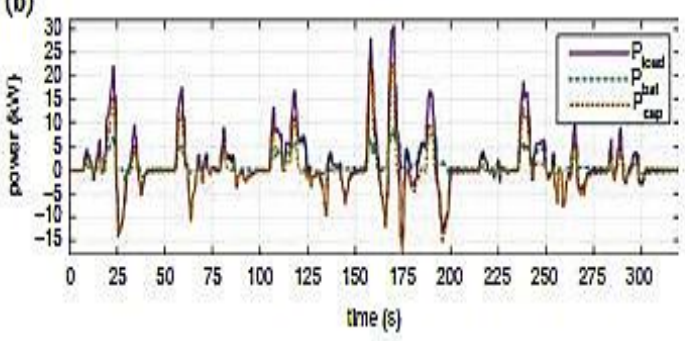

Figure11- (a) Speed and (b) power division drive cycle.

\subsection{Simulation Results}

The forward simulation model is built up using MATLAB/SIMULINK environment to confirm the impact of different shapes of membership functions used in fuzzy logic controller as shown in Figure 12. This simulation contains a Plug-in hybrid-electric vehicle (PHEV) model built using Simscape, Simscape Electrical, and Simscape Driveline, that can be configured for system-level tests or power quality analysis. Model variants for the electrical, battery, and vehicle dynamics systems which can be selected using variant subsystems. A battery model created with the Simscape language is incorporated into the model. Supervisory logic is implemented with Stateflow. This model can be configured for hardware- in-the-loop testing [8], [18].

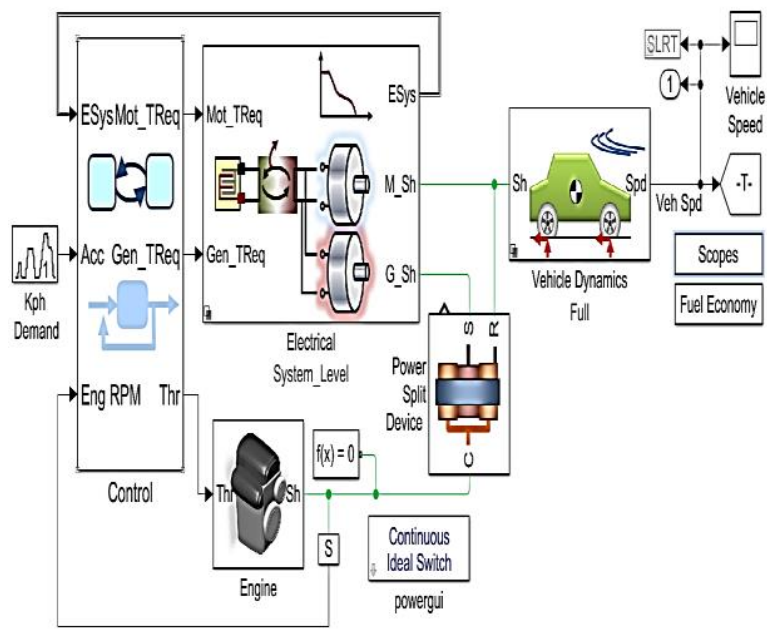

Figure 12- PHEV Simulation Model.
The relation between Speed of PHEV and the Power after using Fuzzy logic controller are shown in Figures 13 and 14.

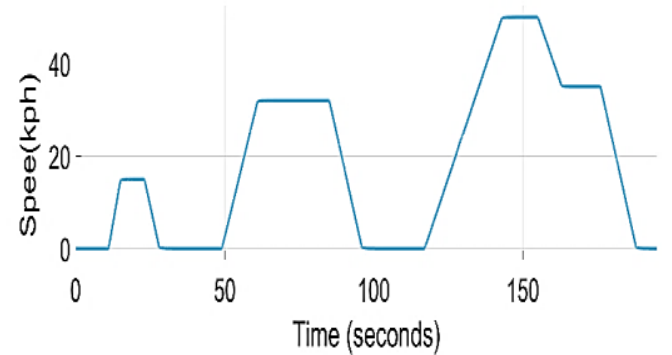

Figure 13- Speed of The Car.

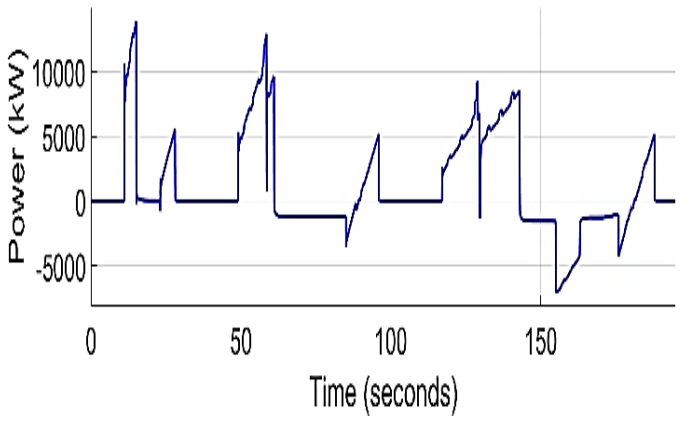

Figure 14- Power of The Car.

\section{Optimal Power Management Algorithm}

The hybrid energy storage requires a power management, which ensures proper energy flow from and to ultracapacitors (UCs), especially when UCs do not store energy required to accelerate the maximum speed. Too early support by UCs may cause empty storage and the lack of support in the final stage of the acceleration when the load power is the highest. On the other hand, focusing only on the highest power pulses results in a rare and not efficient use of UCs. To highlight this case, referring to the fact that keeping a high UCs state of charge (SOC) at medium speed that does not allow to recover energy to the UCs in case of braking. The fuzzy-logic controller (FLC) is developed for the power management of the hybrid source. The FLC differs from classical control for this kind of hybrid energy storage system (HESS) in convenience of designing. The FLC is able to easily model of complex system behavior using expert knowledge-based rules.

A schematic representation of the control strategy is shown in Figure 15. There are two inner current control loops associated with two DC/DC converters. Maintaining desirable output voltage of the hybrid source along with the voltage of UCs as well as appropriate power partitioning between the two energy sources is achieved by a proper reference current value determination by means of FLC [17], [18]. 


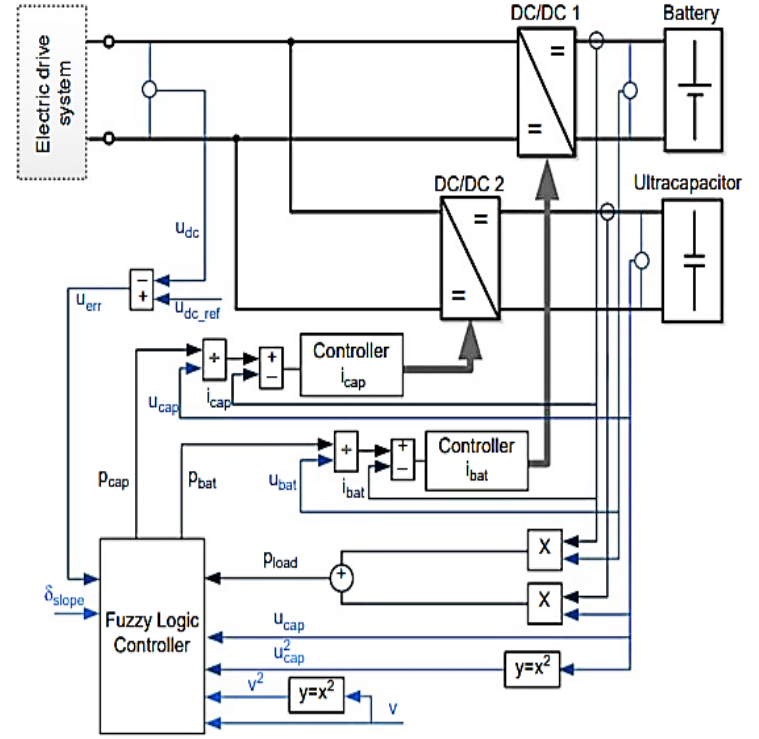

Figure-15 Control scheme for the hybrid energy storage.

Table 2 shows a comparison between the initial EVs and EVs with FLC in terms of Engine system, including the power, minimum speed, maximum speed, and torque of EVs which shows that the FLC improves the EV's performances.

Table 3 shows a comparison between the internal combustion engine (ICEs) in EVs and fuel consumption in EVs with FLC which is decreased after using the fuzzy logic controller.

Table 4 shows the number of batteries which is decreased for EVs after using a fuzzy logic controller compared to the system before using a fuzzy logic controller. Moreover, the decreasing number of the batteries leads to decrease the overall cost of the system.

Table 2- Engine System Requirements

\begin{tabular}{|c|c|c|}
\hline \multicolumn{3}{|c|}{ Engine System } \\
\hline Term & Before FLC & After FLC \\
\hline Power & $\begin{array}{c}57 \mathrm{~kW} \mathrm{@} \\
5000 \mathrm{RPM}\end{array}$ & $\begin{array}{c}86 \mathrm{~kW} @ 5000 \\
\text { RPM }\end{array}$ \\
\hline Min speed & $1000 \mathrm{RPM}$ & $1800 \mathrm{RPM}$ \\
\hline Max speed & $4500 \mathrm{RPM}$ & $5200 \mathrm{RPM}$ \\
\hline Torque & $\begin{array}{c}115 \mathrm{~N} . \mathrm{m} @ \\
4200 \mathrm{RPM}\end{array}$ & $\begin{array}{c}174 \text { N.m @ } \\
4200 \mathrm{RPM}\end{array}$ \\
& & \\
\hline
\end{tabular}

Table 3- Fuel Consumption of EVs

\begin{tabular}{|c|c|}
\hline \multicolumn{2}{|c|}{ Fuel Consumption } \\
\hline Before FLC & $95 \mathrm{MPG}$ \\
\hline After FLC & $51 \mathrm{MPG}$ \\
\hline
\end{tabular}

Table 4- Number of Batteries

\begin{tabular}{|c|c|}
\hline \multicolumn{2}{|c|}{ Number of Batteries } \\
\hline Before FLC & 5 \\
\hline After FLC & 3 \\
\hline
\end{tabular}

\section{Conclusions}

The aim of this paper is to combine hybrid power of super capacitor and battery for hybrid electric vehicles. Design of the power management control, and a comparative analysis between the two configurations have been discussed for EVs. The battery-ultra capacitor energy storage has been presented. It has been shown that, comparatively limited auxiliary storage of energy can effectively help electrochemical batteries when the typographic data are used. The fuzzy logic controller strategy for hybrid energy storage and the use of the physical simulator have been planned and scrutinized in a simulation of the hybrid electric vehicles.

In this paper, the optimal design of energy management system strategies has been conducted using a fuzzy logic controller with different degrees of membership functions. The FLC can effectively distribute the power flow between the battery and ultra-capacitor in the system. Membership function shapes and distribution slightly affect the performance of power flow between the battery and ultracapacitor. In this simulation, the triangular membership function has been decided for the system since it can compensate the power flow performance.

Through the optimal management of the multiple hybrid electric vehicle's operation, it is possible to reduce the number of batteries used in the systems integrated with smart grid. Also, the consumption of the batteries has been reduced. Thus, the overall cost of the EVs have been reduced.

\section{References}

[1] Sami, I., et al. (2019), "A bidirectional interactive electric vehicles operation modes: Vehicle-togrid (V2G) and grid-to-vehicle (G2V) variations within smart grid", 2019 International Conference on Engineering and Emerging Technologies (ICEET), IEEE.

[2] Sabri, M., et al. (2016), "A review on hybrid electric vehicles architecture and energy management strategies" , 53: 1433-1442), IEEE.

[3] Nikowitz, Michael, "Advanced hybrid and electric vehicles" , System Optimization and Vehicle Integration, Springer (2016), IEEE.

[4] Bilgin, B., et al . (2015), "Making the case for electrified transportation", 1(1): 4-17,IEEE. 
[5] Anselma, P. G., et al. capacitor, "Comparing Parallel Hybrid Electric Vehicle Powertrains for Real-world Driving, 2019 AEIT International Conference of Electrical and Electronic Technologies for Automotive (Aeit Automotive)",(2019), IEEE.

[6] Serrao, L., et al., "A comparative analysis of energy management strategies for hybrid electric vehicles" 133(3),(2011),IEEE.

[7] Xiong, R., et al., "Reinforcement learning-based real-time power management for hybrid energy storage system in the plug-in hybrid electric vehicle" , 211: 538-548,(2018), IEEE.

[8] Essoufi, M., B. Hajji, and A. Rabhi, "Fuzzy Logic based Energy Management Strategy for Fuel Cell Hybrid Electric Vehicle", 2020 International Conference on Electrical and Information Technologies (ICEIT),(2020), IEEE.

[9] Mombeshora, Edwin T., and Vincent O. Nyamori ", A review on the use of carbon nanostructured materials in electrochemical capacitors", International Journal of Energy Research 39.15 (2015): 1955-1980, IEEE.

[10] Xiong, Rui, Jiayi Cao, and Quanqing $\mathrm{Yu}$,"Reinforcement learning-based real-time power management for hybrid energy storage system in the plug-in hybrid electric vehicle", Applied energy 211 (2018): 538-548, IEEE.

[11] Morgan, Trevor, "Smart grids and electric vehicles: Made for each other" , International Transport Forum Discussion Paper, 2014 , IEEE.

[12] Briones, A., J. Francfort, P. Heitmann, M. Schey, S. Schey, and J.Smart, "Vehicle-to-grid (V2G) power flow regulations and building codes review by the AVTA", Idaho National Lab, Idaho Falls, ID, USA (2017), IEEE.

[13] Better Air Creating eco-friendly vehicles for the world. Available:[online]

https://www.toyotaeurope.com/worldoftoyota/fe el/environment/better-air/betterair.json.

[14] Yu, Yue, Onyema S. Nduka, and Bikash C. Pal,"Smart Control of an Electric Vehicle for Ancillary Service in DC Microgrid ", IEEE Access (2020).
[15] Gowri, Krishnan, Richard M. Pratt, Francis K. Tuffner, and Michael CW Kintner-Meyer, "Vehicle to Grid Communication Standards Development, Testing and Validation-Status Report", No. PNNL-20844. Pacific Northwest National Laboratory (PNNL), Richland, WA(US), 2011,IEEE.

[16] Kan MiaoTan, Vigna K. Ramachandaramurthy, Jia Ying Yong," Integration of electric vehicles in smart grid: A review on vehicle to grid technologies and optimization techniques", Renewable and Sustainable Energy Reviews 53(2016)720-732.

[17] Masmoudi, Ahmed, et al, "Fuzzy logic based power management strategy using topographic data for an electric vehicle with a batteryultracapacitor energy storage" , COMPEL: The International Journal for Computation and Mathematics in Electrical and Electronic Engineering (2015), IEEE.

[18] Mahyiddin, S. H., et al, "Fuzzy logic energy management system of series hybrid electric vehicle." (2016): 10-6, IEEE. 